\title{
Reversible Trapping of Colloids in Microgrooved Channels via Diffusiophoresis under Steady-State Solute Gradients
}

\author{
Naval Singh $\odot,{ }^{1}$ Goran T. Vladisavljević $\odot,{ }^{1}$ François Nadal, ${ }^{2}$ Cécile Cottin-Bizonne $\odot,{ }^{3}$ \\ Christophe Pirat, ${ }^{3}$ and Guido Bolognesi ${ }^{1, *}$ \\ ${ }^{1}$ Department of Chemical Engineering, Loughborough University, Loughborough LE11 3TU, United Kingdom \\ ${ }^{2}$ Wolfson School of Mechanical, Electrical and Manufacturing Engineering, Loughborough University, \\ Loughborough LE11 3TU, United Kingdom \\ ${ }^{3}$ Institut Lumière Matière, UMR5306 Université Claude Bernard Lyon 1-CNRS, Université de Lyon, \\ Villeurbanne Cedex 69622, France
}

(Received 21 July 2020; revised 24 October 2020; accepted 18 November 2020; published 11 December 2020)

\begin{abstract}
The controlled transport of colloids in dead-end structures is a key capability that can enable a wide range of applications, such as biochemical analysis, drug delivery, and underground oil recovery. This Letter presents a new trapping mechanism that allows the fast (i.e., within a few minutes) and reversible accumulation of submicron particles within dead-end microgrooves by means of parallel streams with different salinity level. For the first time, particle focusing in dead-end structures is achieved under steadystate gradients. Confocal microscopy analysis and numerical investigations show that the particles are trapped at a flow recirculation region within the grooves due to a combination of diffusiophoresis transport and hydrodynamic effects. Counterintuitively, the particle velocity at the focusing point is not vanishing and, hence, the particles are continuously transported in and out of the focusing point. The accumulation process is also reversible and one can cyclically trap and release the colloids by controlling the salt concentration of the streams via a flow switching valve.
\end{abstract}

DOI: 10.1103/PhysRevLett.125.248002

Particle transport in confined structures plays an important role in several technological applications, including drug delivery, diagnostics, enhanced oil recovery, particle separation, and filtration technologies. Nevertheless, the implementation of an effective strategy for controlling the motion of colloidal particles within a confined environment, such as a dead-end channel or a porous medium, is still a challenging and thought-provoking task. In recent years, an increasing number of studies have exploited the motion of particles and liquids induced by solute concentration gradients - the so-called diffusiophoresis (DP) and diffusio-osmosis (DO) phenomena - to enable particle manipulation capabilities, such as delivery to and extraction from dead-end pores [1-3], particle focusing [4-8], and separation [9-11]. In DP with electrolytes, the motion of a particle is driven by a solute concentration gradient $\nabla c$ and the resulting particle velocity can be expressed as $\mathbf{u}_{\mathrm{DP}}=\Gamma_{\mathrm{DP}} \nabla \ln c$, with the DP coefficient $\Gamma_{\mathrm{DP}}$ being a function of the particle and solution properties [12]. Externally applied unsteady solute gradients have been

Published by the American Physical Society under the terms of the Creative Commons Attribution 4.0 International license. Further distribution of this work must maintain attribution to the author(s) and the published article's title, journal citation, and DOI. adopted to boost the otherwise slow and diffusion-limited migration of nano- or microparticles within dead-end structures [1-3]. However, due to the transient nature of the imposed gradients, the particle and flow manipulation capabilities are lost within a short period of time (typically, a few tens of minutes). The ability to generate a steady-state solute gradient within dead-end structures and, hence, retain indefinitely the particle manipulation capability has yet to be achieved. On the other hand, steady-state solute gradients have been used to accumulate colloids in target locations within microfluidic chambers and openended microchannels. For instance, steady-state gradients of chemically reactive solutes [6] can induce particle focusing at the location where $\mathbf{u}_{\mathrm{DP}}=0$. Alternatively, particle focusing can be achieved also by counteracting the DP particle migration with a hydrodynamic flow, $\mathbf{u}$, that advects the particles in the opposite direction $[7,8,11]$. As a result, particle accumulation occurs at the regions where the particle's total velocity, $\mathbf{u}_{p}=\mathbf{u}+\mathbf{u}_{\mathrm{DP}}$, vanishes. For submicron particles, however, their slow Brownian diffusion cannot compete with the particle velocity $\mathbf{u}_{p}$ and, thus, the particle concentration increases indefinitely until the packing limit is reached and the microchannels are irreversibly clogged [7].

Here, we report a new focusing mechanism through which submicron particles can be rapidly and reversibly accumulated within dead-end structures (microgrooves) by 


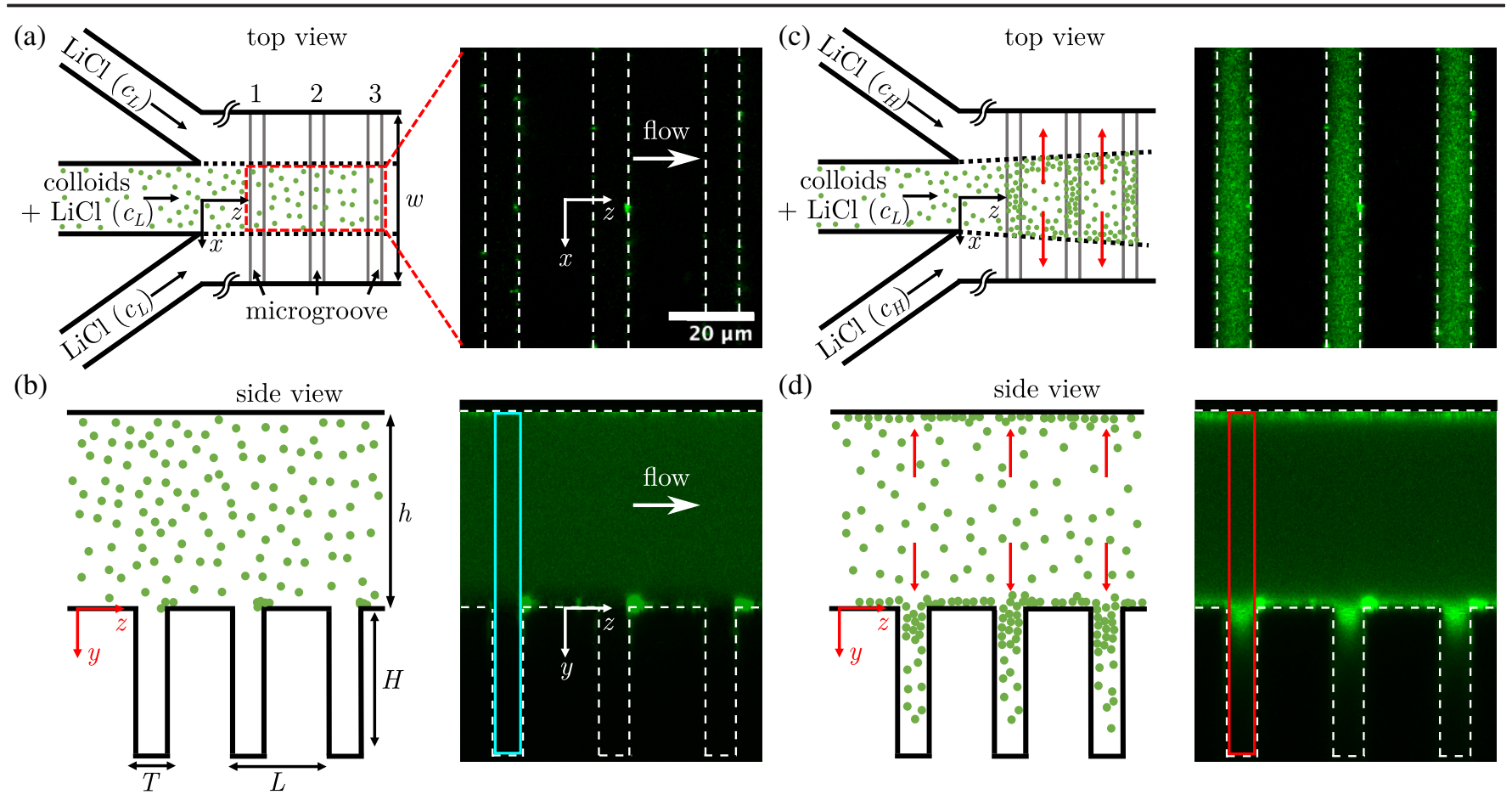

FIG. 1. Solute-induced particle trapping in microgrooves under steady-state conditions. (a),(b) Schematics of top and side view of the device and corresponding fluorescence micrographs of three grooves located at $4 \mathrm{~mm}$ from the junction without solute contrast. Outer flow: $\mathrm{LiCl}$ in DI water at low concentration, $c_{L}=0.1 \mathrm{mM}$. Inner flow: colloids $(215 \mathrm{~nm}$ in diameter @ $0.025 \% \mathrm{v} / \mathrm{v})+\mathrm{LiCl}$ in DI water at low concentration, $c_{L}$. (c),(d) Schematics and micrographs as in panels (a) and (b) with solute contrast. Outer flow: LiCl in DI water at high concentration, $c_{H}=10 \mathrm{mM}$. Inner flow: colloids $+\mathrm{LiCl}$ in DI water at low concentration, $c_{L}$. Red arrows show the direction of the salt gradient. White dashed lines represent channel boundaries and groove edges. Channel size: width $w=400$, depth $h=57 \mu \mathrm{m}$. Groove size: thickness $T=8$, depth $H=45$, pitch $L=32 \mu \mathrm{m}$. In (a) and (c), fluorescence intensities are averaged along the channel depth direction $y$, whereas in (b) and (d) intensities are averaged over the width direction $x$. The same color scale applies to all micrographs. Blue and red rectangles show the integration windows over which the particle concentration profiles in Fig. 2 are calculated.

means of a steady-state gradient. In contrast with other focusing strategies, a steady-state particle distribution is achieved within a few minutes; meanwhile, the concentration peak remains well below the packing limit, thereby avoiding irreversible effects such as particle clustering and device clogging. The particle accumulation process is hence fully reversible and colloids can be transported into and out of the grooves multiple times by switching between different flow streams.

To create a steady-state solute gradient, parallel flows are injected into a $\Psi$-shaped microchannel, made of an optical adhesive (NOA81) glued on top of a silicon substrate with transverse microgrooves, as schematically shown in Fig. 1(a)-see Supplemental Material for details on the device fabrication [13]. A total of 1250 grooves are evenly distributed along the $4 \mathrm{~cm}$ length of the device. The inner flow is a suspension of carboxylate polystyrene fluorescent colloids (Fluoresbrite ${ }^{\circledR} \mathrm{YG}, 0.20 \mu \mathrm{m}$, Polysciences) at a concentration of $n_{0}=0.025 \% \mathrm{v} / \mathrm{v}$, dispersed in a water (Ultrapure Milli-Q) solution of $\mathrm{LiCl}$ (Acros Organics, 99\%) at low concentration, $c_{L}=0.1 \mathrm{mM}$. The outer flow is a $\mathrm{LiCl}$ solution at either low concentration $c_{L}$
[Figs. 1(a)-1(b)] or high concentration $c_{H}=10 \mathrm{mM}$ [Figs. 1(c)-1(d)]. Both the inner and outer flow rates are equal to $12.5 \mu \mathrm{L} / \mathrm{min}$, resulting in an average speed $U_{0}$ of $18.3 \mathrm{~mm} / \mathrm{s}$. The 3D distribution of particles in the channel and the grooves is measured via laser scanning confocal microscopy as detailed in the Supplemental Material [13]. In the absence of salt contrast, the colloidal particles hardly penetrate the grooves likely due to steric and electrokinetic wall-exclusion effects $[2,18]$, which keep most of the particles away from the grooved substrate [Fig. 1(b)]. In the presence of a salt contrast, the particles are expected to migrate towards a region at a higher salt concentration, since the DP coefficient of the particles, $\Gamma_{\mathrm{DP}}$, is positive (see Supplemental Material [13] for the evaluation of $\Gamma_{\mathrm{DP}}$ ). A higher salt concentration in the outer flow streams generates a solute gradient along the channel width direction ( $x$ axis) — see red arrows in Fig. 1(c)—which leads to colloid spreading along the same direction, as also previously reported in a similar flow configuration [4]. More interestingly, salt gradients arise also along the depth direction ( $y$ axis) — see red arrows in Fig. 1(d)—-thereby dragging the particles towards the channel's top flat wall 


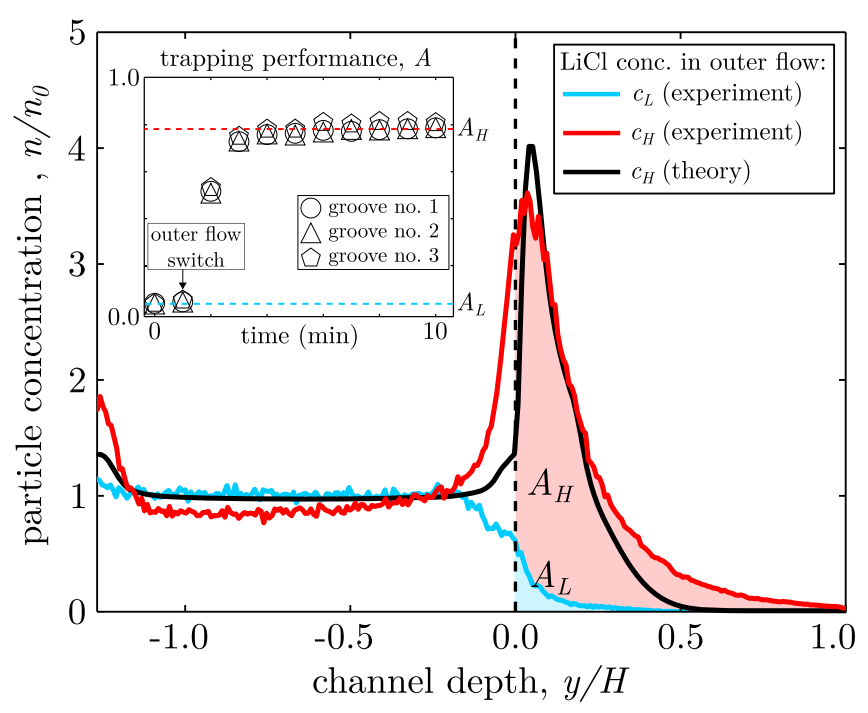

FIG. 2. Experimental steady-state particle concentration profiles along the channel depth without salt gradient (blue curve) and with salt gradient (red curve), corresponding to the blue and red integration windows in Figs. 1(b) and 1(d), respectively. $A_{L}$ and $\mathrm{A}_{H}$ are the integral of the profiles within the groove $(y \geq 0)$ without and with salt gradient, respectively. The profile (black curve), predicted by the numerical simulation in the presence of salt gradient, is also shown. Inset: trapping performance of three neighboring grooves, at $4 \mathrm{~mm}$ from the junction, as the outer flow is switched from low $\left(c_{L}=0.1\right)$ to high $\left(c_{H}=10 \mathrm{mM}\right)$ salt concentration.

and inside the grooves. The $y$ component of the salt gradient is originated by the Poiseuille-like velocity profile in the rectangular channel. Indeed at distances $z$ from the junction much smaller than $U_{0} h^{2} / D_{s} \simeq 4 \mathrm{~cm}$-with $D_{s}$ the salt diffusivity - the salt diffusion process is affected by the nonuniform velocity profile along the channel depth, and the width of the salt diffusive zone at the interface between the inner and outer flows decreases with the distance from the top and bottom walls [19]. Consequently, in the inner region of the channel (i.e., $|x| / w<0.5$ ) a solute gradient directed from the bulk towards the walls is established. It is worth noting that the DP migration of charged particles towards the channel walls in a parallel flow configuration has never been reported before and it could be exploited as a charge-based particle focusing-filtration strategy in microdevices with flat walls only. Driven by DP, the particles migrate towards the grooves and accumulate at the groove's entrance as shown in Figs. 1(c)-1(d).

Figure 2 shows the steady-state colloid concentration profiles along the depth direction, with salt gradient (red line) and without salt gradient (blue line), for groove 1 in Fig. 1. The 3D colloid concentration field $n(x, y, z)$ are calculated from the fluorescence intensity of the confocal scan images via a calibration curve (see Fig. S2 [13]). The concentration profiles in Fig. 2 are calculated for each groove by averaging $n(x, y, z)$ over the $x$ range of the confocal images (ca. $x / w \in[-0.2,0.2]$ ) and over the $z$ range corresponding to the groove thickness $T$, as highlighted by the solid rectangles in the side-view micrographs shown in Figs. 1(b), 1(d). As shown in Fig. 2, the salt contrast between the parallel streams induces the particle migration from the channel bulk towards the top flat wall $(y \rightarrow-h)$ and the groove $(y>0)$, whereas a slight decrease of particle concentration in the bulk $\left(n<n_{0}\right)$ is observed. By definition, the area $A$ below the profile curves for $y>0$ (shaded regions) corresponds to the average particle concentration within the groove, normalized with respect to $n_{0}$. The parameter $A$ can be hence used as a measure of the groove trapping performance. The inset in Fig. 2 shows the evolution of the trapping performance $A$ for the three consecutive grooves shown in Fig. 1, as the outer flow is switched, at the arbitrary time $t=1 \mathrm{~min}$, from low $\left(c_{L}\right)$ to high $\left(c_{H}\right)$ salt concentration solution by means of a flow switching valve. In a few minutes, the value of $A$ increases from $A_{L}=0.022 \pm 0.002$ to $A_{H}=$ $0.79 \pm 0.03$ at steady state, thereby resulting in ca. 36-fold increase in the average particle concentration within the grooves.

To shed light on the mechanisms governing the particle trapping, a numerical analysis (see Supplemental Material [13] for details) is performed in COMSOL Multiphysics to simulate the particle concentration field. The 3D computational domain consists of a rectangular channel and a single groove at $4 \mathrm{~mm}$ from the junction. A slip velocity, $\mathbf{u}_{s}=-\Gamma_{\mathrm{DO}} \nabla \ln c$, with $\Gamma_{\mathrm{DO}}$ the diffusio-osmosis coefficient, is imposed at the domain walls whereas the velocity of the particles $\mathbf{u}_{p}$ is defined as the sum of the hydrodynamic velocity and DP velocity, $\mathbf{u}_{p}=\mathbf{u}+\mathbf{u}_{\mathrm{DP}}$. The value of $\Gamma_{\mathrm{DO}}$ for the channel walls could not be measured so this parameter is adjusted in order to achieve a good match between experimental and numerical results (see Supplemental Material [13]). The colloid concentration profile along the channel depth predicted by the numerical simulation - shown as a black curve in Fig. 2-compares well with the experimental profile (red curve). The good agreement between experiments and theoretical predictions is confirmed also by the experimental and simulated particle concentration field on a plane perpendicular to the flow direction, shown in Figs. 3(a) and 3(b), respectively. These cross-section views show that the salt gradient leads to the accumulation of particles just below the groove entrance as well as the formation of two symmetric and weaker focusing regions, nearly $0.2 w(\simeq 80 \mu \mathrm{m})$ apart from each other, close the top flat wall (see insets). Indeed, in the region $|x| / w<0.5$, the particles are transported towards the outer flow as well as the top and bottom walls by the DP velocity field $\mathbf{u}_{\mathrm{DP}}$, shown in Fig. 4(a). As anticipated, in the channel the salt concentration isolines, also shown in Fig. 4(a), are bent towards the outer flow due to the Poiseuille-like hydrodynamic velocity profile. To clarify why particles accumulate only at the groove entrance 


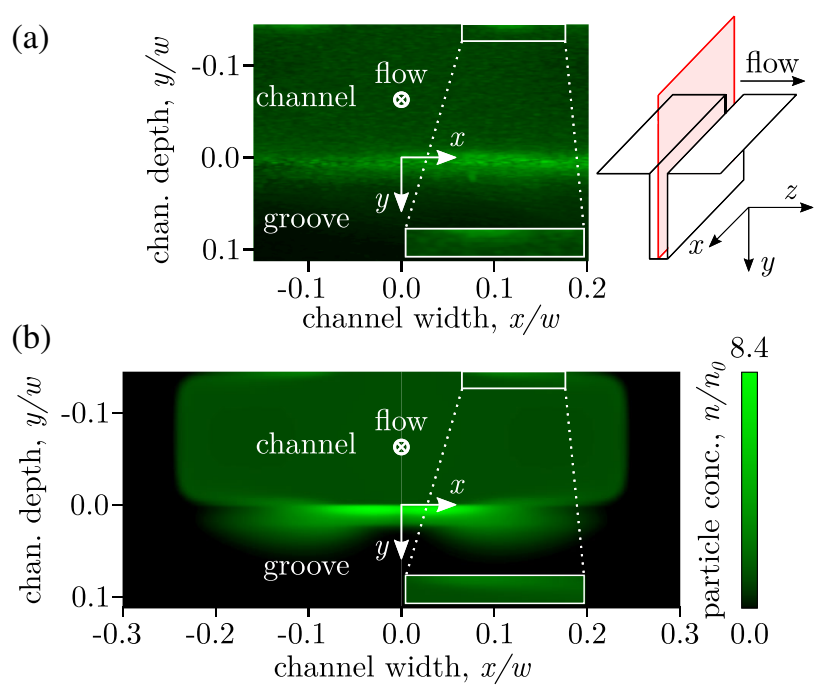

FIG. 3. Experimental (a) and simulated (b) steady-state particle distribution on a plane perpendicular to the flow direction, corresponding to the red-shaded region in the cartoon, at $4 \mathrm{~mm}$ from the junction and with a solute gradient $\left(c_{L}=0.1\right.$ and $c_{H}=10 \mathrm{mM}$ ). Fluorescence intensities (a) and simulated particle concentration (b) are averaged over the groove thickness $T$ (along the $z$ axis). The close-ups highlight one of the two focusing regions near the top flat wall.

without traveling further deep, one should look at the particle distribution together with the streamlines of the velocity field $\mathbf{u}_{p}$ at a cross section perpendicular to the channel widthwise direction. Figure 4(b) shows such a plot for the $y-z$ cross section, $x / w=0.03$, where the maximum particle concentration is achieved $\left(n_{\max } / n_{0} \simeq 15\right)$. It can be seen that the hydrodynamic field $\mathbf{u}$ is characterized by a recirculation region at the groove entrance and a DO-induced flow with opposite direction with respect to the particle DP velocity, i.e., outwards of the groove. Therefore, as the particles migrate towards the groove by DP, they are captured by the closed flow streamlines in the recirculation region and accumulate at the center of the recirculation pattern where the in-plane ( $y$ and $z$ ) components of $\mathbf{u}_{p}$ vanish. However, the DP migration further down the groove is counteracted by the DO flow in the opposite direction. Importantly, in the absence of DO, the particles would still accumulate within the recirculation region, but they would also concentrate at the bottom end of the groove due to DP transport (Fig. S4 [13]). It can be concluded that the observed particle trapping is due to the combined effects of DP particle migration and hydrodynamic flow recirculation within the groove. Despite both DO and Brownian diffusion affects the intensity of the particle concentration peak (i.e., $n_{\max } / n_{0}$ ), they are not required to achieve particle trapping at the groove entrance. Most interestingly, the out-of-plane $(x)$ component of particle velocity, $u_{p, x}$ at the examined $y-z$ cross section is nonzero everywhere in the groove, as shown by Fig. 4(c). Consequently, the particles accumulate at a focusing point (a)
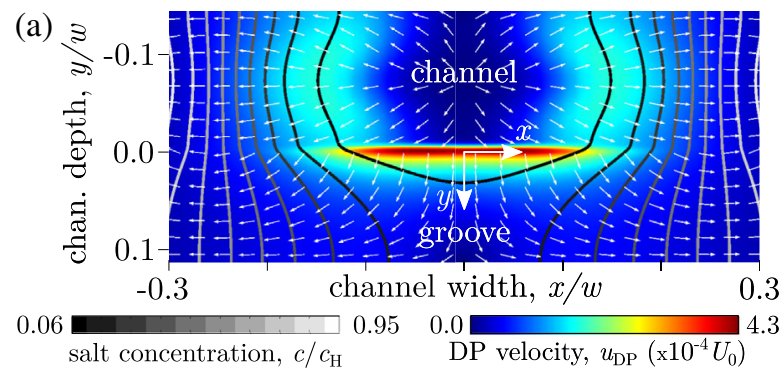

(b)

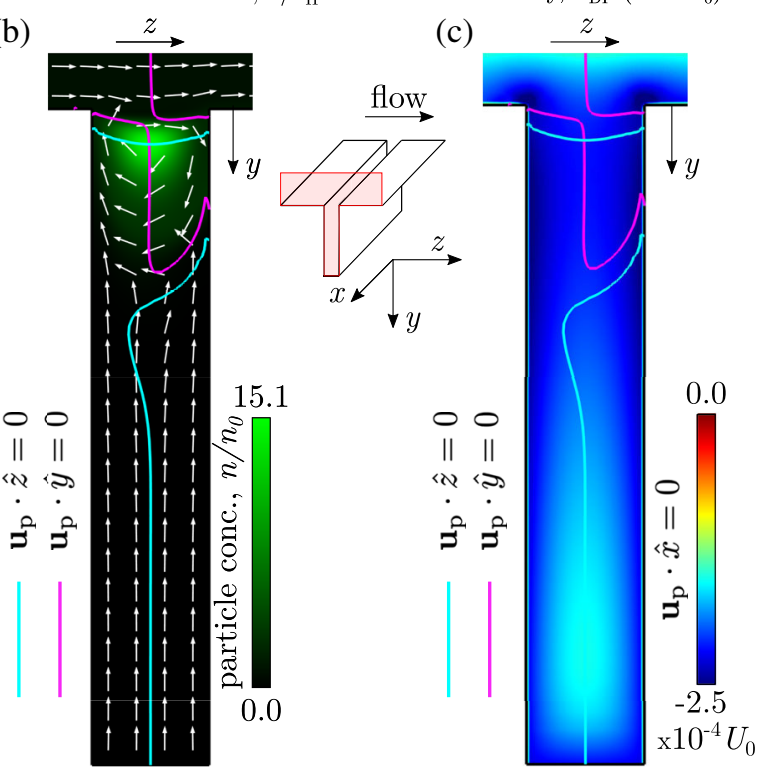

FIG. 4. Numerical simulation results. (a) DP velocity intensity and streamlines, and salt concentration isolines at the same $x-y$ cross section as in Fig. 3. (b) Particle concentration and streamlines of the particle velocity $\mathbf{u}_{p}$ at $y$ - $z$ cross section, $x / w=0.03$, corresponding to the red-shaded region in the cartoon. (c) Out-ofplane component of $\mathbf{u}_{p}$ at the same cross section of panel (b). The blue (magenta) solid lines show the points where the $z$ component ( $y$ component) of $\mathbf{u}_{p}$ vanishes.

where the total particle velocity $\mathbf{u}_{p}$ is nonvanishing and, thus, they are continuously transported in and out of the peak region. The 3D streamlines of the particle velocity field can be seen in Fig. S3 [13]. It is worth noting that $\mathbf{u}_{p}$ vanishes at the center of the flow recirculation at the $y-z$ cross section $x=0$, since $u_{p, x}=0$ due to symmetry. Counterintuitively, in the examined system the particle concentration peak is not achieved at that position despite $\mathbf{u}_{p}=0$ and $\nabla \cdot \mathbf{u}_{p}<0$. Note that the latter relation is a necessary condition for particle focusing (see derivation in the Supplemental Material [13]), whereas the former is neither necessary nor sufficient for focusing to occur.

Our physical interpretation of the trapping mechanism is validated also by the fact that the simulation predicts a location of the peak in the particle concentration profile along the channel depth (Fig. 2) at $y / H=0.052$, which agrees very well with the peak location observed in the experiments (i.e., $y / H=0.051 \pm 0.003$ ). Furthermore, the trapping efficiency, $A=0.69$, in the simulations 


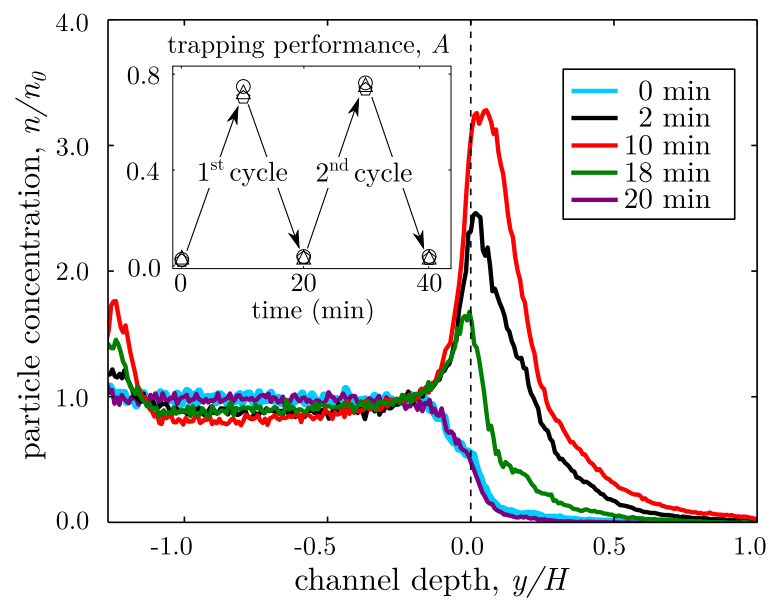

FIG. 5. Reversibility of the particle trapping phenomenon. Time evolution of particle concentration profiles as the colloids are delivered and extracted from the groove by alternating the outer flow between the $\mathrm{LiCl}$ solutions at $c_{H}=10$ and $c_{L}=0.1 \mathrm{mM}$. Inset: trapping performance of three neighboring grooves during two delivery and extraction cycles.

compares well with the one calculated from experiments $(A=0.79 \pm 0.03)$.

Upon removal of the salt gradient, the trapping mechanism ceases and the particles can freely diffuse out of the grooves. Such an effect allows one to control the delivery and extraction of particles into and from the grooves by simply adjusting the salinity contrast between the inner and outer flows. This capability is confirmed by the experimental results, shown in Fig. 5, where the outer flow is alternated between two $\mathrm{LiCl}$ solutions of $c_{L}=0.1$ and $c_{H}=10 \mathrm{mM}$. As the flow configuration is cycled between iso-osmotic $(t=0,20,40 \mathrm{~min})$ and salt gradient $(t=10$, $30 \mathrm{~min}$ ) conditions, the colloid concentration profile and the groove trapping performance change accordingly over time and return to the initial values at the end of each cycle.

To conclude, this Letter demonstrates a new mechanism for reversible trapping of submicron particles in dead-end geometries under steady-state gradients. The key ingredients, enabling the fast and steady accumulation of colloids, are the diffusiophoresis migration of particles along the groove depth direction and the flow recirculation region below the groove entrance. Also, the nonvanishing particle velocity in the focusing region prevents submicron particles from clustering and permanently clogging the grooves. As a result, the trapping phenomenon is fully reversible and particles can be cyclically trapped and released. We envisage that the proposed mechanism can unlock new opportunities for the exploitation of DP transport in soft matter and living systems for drug delivery, synthetic biology, and on-chip diagnostics applications. For example, time-controlled sequential delivery of multiple drugs within dead-end pores could be achieved by switching between flow streams laden with particles having different payloads. On-chip preconcentration of extracellular microvesicles and cells, followed by sample release and off-chip downstream analysis (e.g., flow cytometry, SEM/TEM) could also be explored.

Data available on Loughborough University repository at [20].

This research was supported by the EPSRC New Investigator Award No. EP/S013865/1 (awarded to G. B.) the EPSRC Grant No. EP/M027341/1 and the Santander Mobility Award (awarded to N. S.). We thank R. Fulcrand for help with the microdevice manufacturing. We benefited from access to clean room facilities at Institut des Nanotechnologies de Lyon (INL).

*g.bolognesi@lboro.ac.uk

[1] A. Kar, T.-Y. Chiang, I. Ortiz Rivera, A. Sen, and D. Velegol, Enhanced transport into and out of dead-end pores, ACS Nano 9, 746 (2015).

[2] S. Shin, E. Um, B. Sabass, J. T. Ault, M. Rahimi, P. B. Warren, and H. A. Stone, Size-dependent control of colloid transport via solute gradients in dead-end channels, Proc. Natl. Acad. Sci. U.S.A. 113, 257 (2016).

[3] S. Shin, P. B. Warren, and H. A. Stone, Cleaning by Surfactant Gradients: Particulate Removal from Porous Materials and the Significance of Rinsing in Laundry Detergency, Phys. Rev. Applied 9, 034012 (2018).

[4] B. Abécassis, C. Cottin-Bizonne, C. Ybert, A. Ajdari, and L. Bocquet, Boosting migration of large particles by solute contrasts, Nat. Mater. 7, 785 (2008).

[5] J. Palacci, B. Abécassis, C. Cottin-Bizonne, C. Ybert, and L. Bocquet, Colloidal Motility and Pattern Formation Under Rectified Diffusiophoresis, Phys. Rev. Lett. 104, 138302 (2010).

[6] N. Shi, R. Nery-Azevedo, A. I. Abdel-Fattah, and T. M. Squires, Diffusiophoretic Focusing of Suspended Colloids, Phys. Rev. Lett. 117, 258001 (2016).

[7] S. Shin, J. T. Ault, P. B. Warren, and H. A. Stone, Accumulation of Colloidal Particles in Flow Junctions Induced by Fluid Flow and Diffusiophoresis, Phys. Rev. X 7, 041038 (2017).

[8] S. M. Friedrich, J. M. Burke, K. J. Liu, C. F. Ivory, and T.-H. Wang, Molecular rheotaxis directs dna migration and concentration against a pressure-driven flow, Nat. Commun. 8, 1213 (2017).

[9] S. Shin, O. Shardt, P. B. Warren, and H. A. Stone, Membraneless water filtration using CO2, Nat. Commun. 8, 15181 (2017).

[10] D. Ha, S. Seo, K. Lee, and T. Kim, Dynamic transport control of colloidal particles by repeatable active switching of solute gradients, ACS Nano 13, 12939 (2019).

[11] M. K. Rasmussen, J. N. Pedersen, and R. Marie, Size and surface charge characterization of nanoparticles with a salt gradient, Nat. Commun. 11, 2337 (2020).

[12] D. C. Prieve, J. L. Anderson, J. P. Ebel, and M. E. Lowell, Motion of a particle generated by chemical gradients. Part 2. Electrolytes, J. Fluid Mech. 148, 247 (1984). 
[13] See Supplemental Material at http://link.aps.org/ supplemental/10.1103/PhysRevLett.125.248002 for details on device fabrication, image acquisition and analysis, evaluation of diffusiophoresis coefficient, and numerical simulation. Supplemental Material includes Refs. [14-17].

[14] D. Bartolo, G. Degré, P. Nghe, and V. Studer, Microfluidic stickers, Lab Chip 8, 274 (2008).

[15] K. Tanaka and M. Nomura, Measurements of tracer diffusion coefficients of lithium ions, chloride ions and water in aqueous lithium chloride solutions, J. Chem. Soc., Faraday Trans. 1 83, 1779 (1987).

[16] R. Mills, The self-diffusion of chloride ion in aqueous alkali chloride solutions at 25, J. Phys. Chem. 61, 1631 (1957).
[17] C. Lee, C. Cottin-Bizonne, A.-L. Biance, P. Joseph, L. Bocquet, and C. Ybert, Osmotic Flow Through Fully Permeable Nanochannels, Phys. Rev. Lett. 112, 244501 (2014).

[18] X. Wu, P. Warszynski, and T. Van de Ven, Electrokinetic lift: Observations and comparisons with theories, J. Colloid Interface Sci. 180, 61 (1996).

[19] R. F. Ismagilov, A. D. Stroock, P. J. Kenis, G. Whitesides, and H. A. Stone, Experimental and theoretical scaling laws for transverse diffusive broadening in two-phase laminar flows in microchannels, Appl. Phys. Lett. 76, 2376 (2000).

[20] https://doi.org/10.17028/rd.lboro.13298240. 